\title{
A Basic Framework for Discrete Clifford Analysis
}

\author{
Hennie De Schepper, Frank Sommen, and Liesbet Van de Voorde
}

\section{CONTENTS}

1. Introduction: The Complex Plane

2. A Discrete Dirac Operator: First Attempt

3. A Discrete Dirac Operator: Second Attempt

4. A Special Case: A Factorization of the Star Laplacian

5. A Model for the Forward and Backward Basis Vectors

Acknowledgments

References
2000 AMS Subject Classification: Primary 30G35

Keywords: Clifford analysis, discrete Dirac operator, discrete monogenic function

\begin{abstract}
A basic framework is derived for the development of a higherdimensional discrete function theory in a Clifford algebra context. The concept of a discrete monogenic function is introduced as a proper generalization of the discrete holomorphic, or monodiffric, functions introduced by Isaacs in the 1950s. A concrete model is provided for the definition of the corresponding discrete Dirac operator.
\end{abstract}

\section{INTRODUCTION: THE COMPLEX PLANE}

Clifford analysis (see, e.g., [Brackx et al. 82, Delanghe et al. 92, Gilbert and Murray 91]) is centered on the notion of monogenic functions, i.e., null solutions of the rotationinvariant vector-valued Dirac operator $\partial_{\mathbf{x}}=\sum_{j=1}^{m} e_{j} \partial_{x_{j}}$, where $\left(e_{1}, \ldots, e_{m}\right)$ forms an orthonormal basis for the quadratic space $\mathbb{R}^{0, m}$ underlying the construction of the real Clifford algebra $\mathbb{R}_{0, m}$. It is a popular viewpoint to consider this function theory as a higher-dimensional analogue of the theory of holomorphic functions in the complex plane.

Recently, several authors have shown interest in finding an appropriate framework for the development of discrete counterparts of the basic notions and concepts of Clifford analysis; see, for example, [Guerlebeck and Hommel 02, Guerlebeck and Hommel 01, Faustino et al. 06, Faustino and Kaehler 07a, Faustino et al. 07]. Some of these contributions, however, are explicitly oriented toward the numerical treatment of problems from potential theory and boundary value problems, rather than toward discrete-function-theoretic results; see also [Guerlebeck and Sproessig 90, Guerlebeck and Sproessig 97]. In this paper, we temporarily abandon the path of possible applications in order to focus on the derivation of the fundamental features of a concrete model for a Clifford algebra framework in which discrete Dirac operators and the corresponding discrete function theories can be developed. Having seen the above-mentioned connection between continuous Clifford analysis and complex analysis in the plane, it is only natural to consider first

(c) A K Peters, Ltd. $1058-6458 / 2009 \$ 0.50$ per page Experimental Mathematics 18:4, page 385 
the already existing discrete holomorphic function theories in order to look for similarities. Special attention is paid to the important property of the Dirac operator (respectively the Cauchy-Riemann operator) factorizing the Laplacian.

Discrete mathematics always involves graphs; here, however, we will consider only the simplest of all graphs in Euclidean space, namely the one corresponding to the rectangular $\mathbb{Z}^{m}$ grid. Harmonic functions on $\mathbb{Z}^{m}$ have been studied for over eighty years, and the study of holomorphic functions on $\mathbb{Z}^{2}$ has a history of over sixty years. The pioneer in the latter field, Rufus Isaacs, introduced two difference equations, both of which are discrete counterparts of the Cauchy-Riemann equation in one complex variable.

Definition 1.1. A complex-valued function $f$ defined on $A \subset \mathbb{Z}[i]$ (the Gaussian integers) is called Isaacs holomorphic (or monodiffric of the first kind) in $A$ if for all $z \in A$ such that $z+1$ and $z+i$ also belong to $A$, we have

$$
\frac{f(z+1)-f(z)}{1}=\frac{f(z+i)-f(z)}{i} .
$$

Definition 1.2. A complex-valued function $f$ defined on $A \subset \mathbb{Z}[i]$ is called Ferrand holomorphic (or monodiffric of the second kind) in $A$ if for all $z \in A$ such that $z+1$, $z+i$, and $z+1+i$ also belong to $A$, we have

$$
\frac{f(z+1+i)-f(z)}{1+i}=\frac{f(z+i)-f(z+1)}{i-1} .
$$

Monodiffric functions of the first kind were studied by Isaacs himself [Isaacs 52] and later in [Nakamura and Rosenfeld 97]. Monodiffric functions of the second kind were investigated in [Ferrand 44] and later in [Duffin 56], [Kenyon 00], and [Benjamini and Lovász 02]. Further references can be found in the recent paper [Kiselman 05].

The above definitions of discrete holomorphy may be expressed by means of corresponding discrete CauchyRiemann operators. Definition 1.1 may then be rephrased as follows.

Definition 1.3. A complex-valued function $f$ defined on $A \subset \mathbb{Z}[i]$ is Isaacs holomorphic in $A$ if at all points $z$ such that $z, z+1, z+i \in A$, we have

$$
\operatorname{cr}_{1}[f](z):=f(z+1)+i f(z+i)-(1+i) f(z)=0
$$

or equivalently $\mu_{1} * f=0$, with $\mu_{1}=\delta_{-1}+i \delta_{-i}-(1+i) \delta_{0}$.
Note that this concept of discrete holomorphy is not invariant under rotation by $\frac{\pi}{2}$ or by $\pi$, but behaves well under reflection with respect to the first bisector $z \mapsto i \bar{z}$.

Splitting $f$ into its real and imaginary parts, $f=u+$ $i v$, with $u, v: \mathbb{Z}[i] \rightarrow \mathbb{R}$, we find that the above CauchyRiemann equation (1-1) may be rewritten as the system

$$
\begin{aligned}
& u(z+1)-v(z+i)-u(z)+v(z)=0, \\
& v(z+1)+u(z+i)-u(z)-v(z)=0,
\end{aligned}
$$

or even as

$$
\begin{aligned}
& u(z+1)-u(z)=v(z+i)-v(z), \\
& v(z+1)-v(z)=-(u(z+i)-u(z)),
\end{aligned}
$$

which in terms of the forward differences

$$
\Delta_{1}^{+} g(z)=g(z+1)-g(z), \quad \Delta_{2}^{+} g(z)=g(z+i)-g(z)
$$

reads

$$
\Delta_{1}^{+} u=\Delta_{2}^{+} v, \quad \Delta_{1}^{+} v=-\Delta_{2}^{+} u .
$$

The latter may be considered a direct analogue of the "continuous" Cauchy-Riemann system $\left\{\partial_{x} u=\partial_{y} v\right.$, $\left.\partial_{x} v=-\partial_{y} u\right\}$.

One of the essential features in complex analysis in the plane is the fact that the Cauchy-Riemann operator "factorizes" the Laplace operator, in the sense that the product of the Cauchy-Riemann operator with its complex conjugate equals the Laplacian. A major point of interest in this paper will be to arrive at a similar property in the discrete setting. To this end we first give a proper definition of the discrete Laplace operator as can be found in the literature for an arbitrary connected graph (in the weightless case).

Definition 1.4. Let $g$ be a function defined on the vertices of a connected graph and let $v$ be an arbitrary such vertex. Then the action of the discrete Laplace operator on $g$ at $v$ is defined by

$$
\Delta g(v)=\sum_{w \sim v}(g(w)-g(v))=\sum_{w \sim v} g(w)-\left(\# \mathcal{N}_{v}\right) g(v),
$$

where the notation $w \sim v$ means that there is an edge in the graph under consideration that links the vertex $w$ to $v$, and where $\mathcal{N}_{v}$ stands for the neighborhood of $v$ with respect to the graph, i.e., the set of all points $w \sim v$.

Here, in the case of $\mathbb{Z}[i]$, we have

$$
\overline{\mathrm{Cr}_{1}}[f](z)=f(z+1)-i f(z+i)-(1-i) f(z),
$$


whence

$$
\begin{aligned}
\overline{\mathrm{Cr}_{1}} \mathrm{cr}_{1}[f](z) \\
=(f(z+2)-f(z+1))-(f(z+1)-f(z)) \\
\quad+(f(z+2 i)-f(z+i))-(f(z+i)-f(z)),
\end{aligned}
$$

which does not take the form of a Laplacian on $\mathbb{Z}[i]$, as might be expected, since the symmetry of the graph was broken in the definition of $\mathrm{cr}_{1}$.

A more symmetric Cauchy-Riemann equation is given by

$\mathrm{cr}_{2}[f](z):=f(z+1)-f(z-1)+i f(z+i)-i f(z-i)=0$,

which indeed uses the complete neighborhood of $z$ in the $\mathbb{Z}^{2}$ grid. Observe that $\mathrm{cr}_{2}$ does not correspond literally to the notion of Ferrand holomorphy, since the latter uses a neighborhood of $z$ consisting of the points $z+1, z+i$, and $z+1+i$. However, Ferrand introduced a CauchyRiemann operator involving the points $z+1+i, z-1+i$, $z-1-i$, and $z+1-i$, which may easily be converted into $\mathrm{cr}_{2}$ by rotation and rescaling. Here, the CauchyRiemann equation (1-2) leads to the system

$$
\begin{aligned}
& u(z+1)-u(z-1)-v(z+i)+v(z-i)=0 \\
& v(z+1)+v(z-1)+u(z+i)-u(z-i)=0
\end{aligned}
$$

which can be rewritten as

$$
\begin{aligned}
& (u(z+1)-u(z))+(u(z)-u(z-1)) \\
& \quad=(v(z+i)-v(z))+(v(z)-v(z-i)), \\
& (v(z+1)-v(z))+(v(z)-v(z-1)) \\
& \quad=-[(u(z+i)-u(z))+(u(z)-u(z-i))],
\end{aligned}
$$

or even, now using also the backward differences

$$
\Delta_{1}^{-} g(z)=g(z)-g(z-1), \quad \Delta_{2}^{-} g(z)=g(z)-g(z-i),
$$

as

$$
\begin{aligned}
& \Delta_{1}^{+} u+\Delta_{1}^{-} u=\Delta_{2}^{+} v+\Delta_{2}^{-} v \\
& \Delta_{1}^{+} v+\Delta_{1}^{-} v=-\left(\Delta_{2}^{+} u+\Delta_{2}^{-} u\right)
\end{aligned}
$$

Introducing the total differences

$$
\Delta_{1}=\frac{1}{2}\left(\Delta_{1}^{+}+\Delta_{1}^{-}\right), \quad \Delta_{2}=\frac{1}{2}\left(\Delta_{2}^{+}+\Delta_{2}^{-}\right),
$$

one finally arrives at

$$
\Delta_{1} u=\Delta_{2} v, \quad \Delta_{1} v=-\Delta_{2} u
$$

which again takes an analogous form as the "continuous" Cauchy-Riemann system. With respect to a possible factorization of the Laplace operator one gets

$$
\begin{aligned}
\overline{\mathrm{Cr}_{2}} \mathrm{Cr}_{2}[f](z)= & f(z+2)+f(z-2)+f(z+2 i) \\
& +f(z-2 i)-4 f(z) .
\end{aligned}
$$

In [Kiselman 05] this is considered an acceptable result, i.e., a discrete Laplacian on a kind of enlarged neighborhood of $z$, the phenomenon of enlarging neighborhoods being typical and seemingly unavoidable when one is composing discrete operators. However, we will show below that it is possible to create a discrete setting in which this phenomenon does not occur, i.e., one that respects the original neighborhood of a given point.

\section{A DISCRETE DIRAC OPERATOR: FIRST ATTEMPT}

We wish to extend the ideas described above to a higherdimensional context. To this end, let $\mathbb{R}^{0, m}$ be endowed with a nondegenerate quadratic form of signature $(0, m)$, let $\left(e_{1}, \ldots, e_{m}\right)$ be an orthonormal basis for $\mathbb{R}^{0, m}$, and let $\mathbb{R}_{0, m}$ be the real Clifford algebra constructed over $\mathbb{R}^{0, m}$; see, e.g., [Porteous 95]. The noncommutative multiplication in $\mathbb{R}_{0, m}$ is governed by

$$
e_{j} e_{k}+e_{k} e_{j}=-2 \delta_{j k}, \quad j, k=1, \ldots, m
$$

A basis for $\mathbb{R}_{0, m}$ is obtained by considering for a set $A=$ $\left\{j_{1}, \ldots, j_{h}\right\} \subset\{1, \ldots, m\}$ the element $e_{A}=e_{j_{1}} \cdots e_{j_{h}}$, with $1 \leq j_{1}<j_{2}<\cdots<j_{h} \leq m$. For the empty set $\varnothing$ one puts $e_{\varnothing}=1$, the identity element. Any Clifford number $a$ in $\mathbb{R}_{0, m}$ may thus be written as $a=\sum_{A} e_{A} a_{A}, a_{A} \in$ $\mathbb{R}$, or even as $a=\sum_{k=0}^{m}[a]_{k}$, where $[a]_{k}=\sum_{|A|=k} e_{A} a_{A}$ is the so-called $k$-vector part of $a(k=0,1, \ldots, m)$. In fact, when allowing for complex constants, the same set of generators $\left(e_{1}, \ldots, e_{m}\right)$, still satisfying the anticommutation rules (2-1), also produces the complex Clifford algebra $\mathbb{C}_{m}$, and hence all real Clifford algebras $\mathbb{R}_{p, q}$ of any signature $(p+q=m)$ as well.

The Euclidean space $\mathbb{R}^{0, m}$ is embedded in $\mathbb{R}_{0, m}$ by identifying $\left(x_{1}, \ldots, x_{m}\right)$ with the Clifford vector $\mathbf{x}$ given by $\mathbf{x}=\sum_{j=1}^{m} e_{j} x_{j}$. The multiplication of any two vectors $\mathbf{x}$ and $\mathbf{y}$ is given by $\mathbf{x y}=\mathbf{x} \cdot \mathbf{y}+\mathbf{x} \wedge \mathbf{y}$ with

$$
\begin{aligned}
\mathbf{x} \cdot \mathbf{y} & =-\sum_{j=1}^{m} x_{j} y_{j}=\frac{1}{2}(\mathbf{x} \mathbf{y}+\mathbf{y} \mathbf{x}) \\
\mathbf{x} \wedge \mathbf{y} & =\sum_{i<j} e_{i j}\left(x_{i} y_{j}-x_{j} y_{i}\right)=\frac{1}{2}(\mathbf{x} \mathbf{y}-\mathbf{y} \mathbf{x}),
\end{aligned}
$$

being the scalar-valued dot product (equal to the Euclidean inner product up to a minus sign) and the 
bivector-valued wedge product, respectively. Note that the square of a vector $\mathbf{x}$ is scalar-valued and equals the norm squared up to a minus sign: $\mathbf{x}^{2}=-\langle\mathbf{x}, \mathbf{x}\rangle=-|\mathbf{x}|^{2}$.

Conjugation in $\mathbb{R}_{0, m}$ is defined as the anti-involution for which $\bar{e}_{j}=-e_{j}, j=1, \ldots, m$. In particular, for a vector $\mathbf{x}$ we have $\overline{\mathbf{x}}=-\mathbf{x}$.

The Fourier dual of the vector $\mathbf{x}$ is the vector-valued first-order differential operator

$$
\partial_{\mathbf{x}}=\sum_{j=1}^{m} e_{j} \partial_{x_{j}}
$$

called the Dirac operator. It is precisely this Dirac operator that underlies the notion of monogenicity of a function, a notion that may be considered as the higherdimensional counterpart of holomorphy in the complex plane. A function $f$ defined and differentiable in an open region $\Omega$ of $\mathbb{R}^{m}$ and taking values in $\mathbb{R}_{0, m}$ is called left monogenic in $\Omega$ if $\partial_{\mathbf{x}}[f]=0$. Since the Dirac operator factorizes the Laplacian, $\Delta=-\partial_{\mathbf{x}}^{2}$, monogenicity may also be regarded as a refinement of harmonicity.

Nowadays, this setting is referred to as the orthogonal case, since the fundamental group leaving the Dirac operator $\partial_{\mathbf{x}}$ invariant is the special orthogonal group $\mathrm{SO}(m)$, which is doubly covered by the $\operatorname{Spin}(m)$ group of the Clifford algebra $\mathbb{R}_{0, m}$. For this reason, the Dirac operator is called a rotation-invariant operator. In the present context, we will also refer to this setting as the continuous case, as opposed to the discrete setting treated here.

As announced above, we will consider the graph corresponding to the equidistant grid $\mathbb{Z}^{m}$; thus a Clifford vector $\mathbf{x}$ as introduced above will now show only integer coordinates. For the pointwise discretization of the partial derivatives $\frac{\partial}{\partial x_{j}}$ we then introduce the traditional one-sided forward and backward differences, respectively given by

$$
\begin{aligned}
\Delta_{j}^{+}[f](\mathbf{x}) & =f\left(\ldots, x_{j}+1, \ldots\right)-f\left(\ldots, x_{j}, \ldots\right) \\
& =f\left(\mathbf{x}+e_{j}\right)-f(\mathbf{x}), \quad j=1, \ldots, m,
\end{aligned}
$$

and

$$
\begin{aligned}
\Delta_{j}^{-}[f](\mathbf{x}) & =f\left(\ldots, x_{j}, \ldots\right)-f\left(\ldots, x_{j}-1, \ldots\right) \\
& =f(\mathbf{x})-f\left(\mathbf{x}-e_{j}\right), \quad j=1, \ldots, m,
\end{aligned}
$$

as well as the two-sided total difference given by

$$
\begin{aligned}
\Delta_{j}[f](\mathbf{x}) & =\frac{1}{2}\left(\Delta_{j}^{+}+\Delta_{j}^{-}\right)[f](\mathbf{x}) \\
& =\frac{1}{2}\left(f\left(\mathbf{x}+e_{j}\right)-f\left(\mathbf{x}-e_{j}\right)\right), \quad j=1, \ldots, m .
\end{aligned}
$$

Combining the latter with the orthonormal basis vectors of the Clifford algebra, we arrive at a first possible definition of a discrete Dirac operator.

Definition 2.1. The discrete Dirac operator $\partial$ is the firstorder Clifford-vector-valued difference operator given by

$$
\partial=\sum_{j=1}^{m} e_{j} \Delta_{j} .
$$

On the other hand, in order to introduce a discrete Laplacian, we will revert to Definition 1.4, which now explicitly reads

$$
\begin{aligned}
\Delta[f](\mathbf{x}) & =\sum_{j=1}^{m}\left[\Delta_{j}^{+}[f](\mathbf{x})-\Delta_{j}^{-}[f](\mathbf{x})\right] \\
& =\sum_{j=1}^{m}\left[\left(f\left(\mathbf{x}+e_{j}\right)-f(\mathbf{x})\right)+\left(f\left(\mathbf{x}-e_{j}\right)-f(\mathbf{x})\right)\right] \\
& =\sum_{j=1}^{m}\left[f\left(\mathbf{x}+e_{j}\right)+f\left(\mathbf{x}-e_{j}\right)\right]-2 m f(\mathbf{x})
\end{aligned}
$$

with respect to the $\mathbb{Z}^{m}$ neighborhood of $\mathbf{x}$. This operator is usually called the star Laplacian: it involves the values of the considered function at the midpoints of the faces of the cube with side length equal to 2 , centered at x. Clearly, with respect to that same grid, but changing the graph, other discrete Laplacians may be defined, involving, for example, the function values at the vertices of the cube (the cross Laplacian), or at the midpoints of the "edges." Correspondingly, alternative Dirac operators may be considered as well. This will be the subject of a forthcoming paper, where it will moreover be investigated whether any combination of such discrete Laplacians admits a (unique) factorization in terms of discrete Dirac operators. For now, we restrict ourselves to the star Laplacian (2-3); note that it can also be written as

$$
\Delta[f](\mathbf{x})=\sum_{j=1}^{m} \Delta_{j}^{+} \Delta_{j}^{-}[f](\mathbf{x})=\sum_{j=1}^{m} \Delta_{j}^{-} \Delta_{j}^{+}[f](\mathbf{x}) .
$$

As could be expected from its similarity with the Cauchy-Riemann operator (1-2) above, the discrete Dirac operator (2-2) does not provide us with a true factorization of the star Laplacian. Instead, for the product with its Clifford conjugate we obtain

$\partial \bar{\partial}=-\partial^{2}=\frac{1}{4} \sum_{j=1}^{m}\left[\left(f\left(\mathbf{x}+2 e_{j}\right)+f\left(\mathbf{x}-2 e_{j}\right)\right)-2 f(\mathbf{x})\right]$, 
which, although taking the form of a star Laplacian (up to a factor), clearly shows the phenomenon of the enlarging neighborhoods; we will denote the obtained result by $4 \partial^{2}=-\Delta_{2}$, the subscript 2 referring to the rescaling of the neighborhood.

Inspired by the alternative form (2-4) of the star Laplacian, we will now take into account the forward and the backward differences separately, by means of a forward discrete Dirac operator $\partial^{+}$, given by

$$
\partial^{+}=\sum_{j=1}^{m} e_{j} \Delta_{j}^{+},
$$

and a backward discrete Dirac operator $\partial^{-}$, given by

$$
\partial^{-}=\sum_{j=1}^{m} e_{j} \Delta_{j}^{-}
$$

see also [Faustino and Kaehler 07a]. This leads to a refinement of the original discrete Dirac operator in the sense that $2 \partial=\partial^{+}+\partial^{-}$. Some direct computations yield

$$
\partial^{+} \partial^{-}+\partial^{-} \partial^{+}=-2 \Delta
$$

while

$$
\begin{aligned}
& \left(\partial^{+}\right)^{2}=-\sum_{j=1}^{m}\left[f\left(\mathbf{x}+2 e_{j}\right)-2 f\left(\mathbf{x}+e_{j}\right)+f(\mathbf{x})\right] \\
& \left(\partial^{-}\right)^{2}=-\sum_{j=1}^{m}\left[f(\mathbf{x})-2 f\left(\mathbf{x}-e_{j}\right)+f\left(\mathbf{x}-2 e_{j}\right)\right]
\end{aligned}
$$

or even $\left(\partial^{+}\right)^{2}+\left(\partial^{-}\right)^{2}=-\Delta_{2}+2 \Delta$, in accordance with the earlier result that $4 \partial^{2}=-\Delta_{2}$. Although a nice result, (2-5) cannot be seen as a true factorization of the star Laplacian. As we aim at developing a discrete function theory in which the notion of discrete monogenicity implies discrete harmonicity, we will abandon these attempts and turn to a completely different approach in the next section.

\section{A DISCRETE DIRAC OPERATOR: SECOND ATTEMPT}

The philosophy behind this alternative approach is the following. In passing from the continuous to the discrete setting, partial derivatives have been replaced by partial differences, i.e., differences according to the directions of the Cartesian coordinate axes. Clearly, there is a choice involved, since each axis carries two senses, leading to the forward and the backward differences. In passing to the Dirac operator, in the continuous case each partial derivative is combined with its corresponding basis vector $e_{j}, j=1, \ldots, m$, while in the discrete case, this symmetry is broken and can be maintained only by introducing socalled forward and backward basis vectors, carrying the chosen sense of the partial differences involved.

To this end, we need to embed the Clifford algebra $\mathbb{R}_{0, m}$ into a bigger one, whose underlying vector space has double dimension. In the most general setting, this will be $\mathbb{C}_{2 m}$. So now let us postulate the existence of $2 m$ vectors $e_{j}^{+}$and $e_{j}^{-}, j=1, \ldots, m$, satisfying the following anticommutator relations:

$$
\begin{aligned}
& e_{j}^{+} e_{k}^{+}+e_{k}^{+} e_{j}^{+}=-2 g_{j k}^{+}, \\
& e_{j}^{-} e_{k}^{-}+e_{k}^{-} e_{j}^{-}=-2 g_{j k}^{-}, \\
& e_{j}^{+} e_{k}^{-}+e_{k}^{-} e_{j}^{+}=-2 M_{j k},
\end{aligned}
$$

where the symmetric tensors $\left(g_{j k}^{+}\right),\left(g_{j k}^{-}\right)$and the general tensor $\left(M_{j k}\right)$ determine the corresponding metric; see also [Faustino et al. 07]. We will make three assumptions on this metric, which will significantly reduce the degree of freedom in the choice of metric scalars.

Assumption 3.1. The forward and the backward basis vectors in each particular Cartesian direction add up to the traditional basis vector in that direction, i.e.,

$$
e_{j}^{+}+e_{j}^{-}=e_{j}, \quad j=1, \ldots, m \text {. }
$$

Clearly, this assumption reflects the philosophy behind our new approach sketched above. The Clifford multiplication rules $e_{j} e_{k}+e_{k} e_{j}=-2 \delta_{j k}$ then already induce the following relations between the metric scalars:

$$
g_{j k}^{+}+g_{j k}^{-}+M_{j k}+M_{k j}=\delta_{j k}, \quad j, k=1, \ldots, m .
$$

Assumption 3.2. There are no preferential Cartesian directions, or in other words, all Cartesian directions play the same role in the metric. This assumption may be referred to as the principle of dimensional equivalence and may be seen as a kind of rotational invariance.

On the basis of this second assumption, we may put

$$
\begin{aligned}
& g_{11}^{+}=g_{22}^{+}=\cdots=g_{m m}^{+}=\lambda^{+}, \\
& g_{11}^{-}=g_{22}^{-}=\cdots=g_{m m}^{-}=\lambda^{-},
\end{aligned}
$$

where $g_{j j}^{ \pm}=-\left(e_{j}^{ \pm}\right)^{2}, j=1, \ldots, m$, and

$$
M_{11}=M_{22}=\cdots=M_{m m}=\mu,
$$

where $2 M_{j j}=-\left(e_{j}^{+} e_{j}^{-}+e_{j}^{-} e_{j}^{+}\right), j=1, \ldots, m$. Furthermore, $g_{j k}^{ \pm}$and $M_{j k}$, for $j \neq k$, should also be independent 
of their subscripts, whence we put

$$
g_{j k}^{ \pm}=g^{ \pm}, \quad j, k=1, \ldots, m, \quad j \neq k
$$

and

$$
M_{j k}=M_{k j}=M, \quad j, k=1, \ldots, m, j \neq k .
$$

In combination with (3-1), equations (3-2)-(3-4) yield

$$
\lambda^{+}+\lambda^{-}+2 \mu=1
$$

while $(3-5)-(3-6)$ lead to

$$
g^{+}+g^{-}+2 M=0
$$

Assumption 3.3. The positive and negative orientations of any Cartesian direction play an equivalent role. This assumption may be interpreted as a kind of reflection invariance.

As a consequence, one should have that

$$
\lambda^{+}=\lambda^{-}=\lambda \quad \text { and } \quad g^{+}=g^{-}=g
$$

whence

$$
\lambda+\mu=\frac{1}{2} \quad \text { and } \quad g+M=0
$$

Summarizing, when we take into account Assumptions $3.1-3.3$, the forward and backward basis vectors $e_{j}^{+}$and $e_{j}^{-}, j=1, \ldots, m$, will obey the following multiplication rules:

$$
\begin{aligned}
& e_{j}^{+} e_{k}^{+}+e_{k}^{+} e_{j}^{+}=e_{j}^{-} e_{k}^{-}+e_{k}^{-} e_{j}^{-}=-2 g, j \neq k, \\
& e_{j}^{+} e_{k}^{-}+e_{k}^{-} e_{j}^{+}=2 g, \quad j \neq k, \\
& \left(e_{j}^{+}\right)^{2}=\left(e_{j}^{-}\right)^{2}=-\lambda, \quad j=1, \ldots, m, \\
& e_{j}^{+} e_{j}^{-}+e_{j}^{-} e_{j}^{+}=2 \lambda-1, \quad j=1, \ldots, m .
\end{aligned}
$$

We are now led to a new definition for our discrete Dirac operator. Note that we formally retain the notation of the previous section, which, however, receives another meaning.

Definition 3.4. The discrete Dirac operator $\partial$ is the firstorder Clifford-vector-valued difference operator given by

$$
\partial=\partial^{+}+\partial^{-}
$$

where the forward and backward discrete Dirac operators $\partial^{+}$and $\partial^{-}$are respectively given by

$$
\partial^{+}=\sum_{j=1}^{m} e_{j}^{+} \Delta_{j}^{+} \quad \text { and } \quad \partial^{-}=\sum_{j=1}^{m} e_{j}^{-} \Delta_{j}^{-} .
$$

We directly obtain, using the above multiplication rules,

$$
\begin{gathered}
\partial^{+} \partial^{+}=-\lambda \sum_{j=1}^{m} \Delta_{j}^{+} \Delta_{j}^{+}-2 g \sum_{j<k} \Delta_{j}^{+} \Delta_{k}^{+} \\
\partial^{-} \partial^{-}=-\lambda \sum_{j=1}^{m} \Delta_{j}^{-} \Delta_{j}^{-}-2 g \sum_{j<k} \Delta_{j}^{-} \Delta_{k}^{-}, \\
\partial^{+} \partial^{-}+\partial^{-} \partial^{+} \\
=(2 \lambda-1) \sum_{j=1}^{m} \Delta_{j}^{+} \Delta_{j}^{-}+2 g \sum_{j \neq k} \Delta_{j}^{+} \Delta_{k}^{-},
\end{gathered}
$$

so that

$$
\begin{aligned}
\partial^{2}= & -\lambda \sum_{j=1}^{m}\left(\Delta_{j}^{+} \Delta_{j}^{+}+\Delta_{j}^{-} \Delta_{j}^{-}\right)+(2 \lambda-1) \sum_{j=1}^{m} \Delta_{j}^{+} \Delta_{j}^{-} \\
& +g \sum_{j \neq k}\left(2 \Delta_{j}^{+} \Delta_{k}^{-}-\Delta_{j}^{-} \Delta_{k}^{-}-\Delta_{j}^{+} \Delta_{k}^{+}\right) .
\end{aligned}
$$

From this expression it directly follows that if we want the support of $\partial^{2}$ to remain in the cube with side length 2 centered at $\mathbf{x}$, then we have to impose the isotropy of the forward and backward basis vectors, i.e., we have to put $\lambda=\left(e_{j}^{+}\right)^{2}=\left(e_{j}^{-}\right)^{2}=0$ as in [Faustino et al. 07], whence in our case it follows in addition that $\mu=\frac{1}{2}$, or $\left\{e_{j}^{+}, e_{j}^{-}\right\}:=e_{j}^{+} e_{j}^{-}+e_{j}^{-} e_{j}^{+}=-1, j=1, \ldots, m$, where we have introduced the notation $\{\cdot, \cdot\}$ for the anticommutator. We now have

$$
\begin{aligned}
e_{j}^{+} e_{k}^{+}+e_{k}^{+} e_{j}^{+} & =e_{j}^{-} e_{k}^{-}+e_{k}^{-} e_{j}^{-}=-2 g, j \neq k, \\
e_{j}^{+} e_{k}^{-}+e_{k}^{-} e_{j}^{+} & =2 g, \quad j \neq k, \\
\left(e_{j}^{+}\right)^{2}=\left(e_{j}^{-}\right)^{2} & =0, \quad j=1, \ldots, m, \\
e_{j}^{+} e_{j}^{-}+e_{j}^{-} e_{j}^{+} & =-1, \quad j=1, \ldots, m .
\end{aligned}
$$

These relations completely determine the metric of the underlying $2 m$-dimensional space in terms of one free scalar parameter $g$. Let us investigate whether there are any restrictions on the values of $g$. To this end, we consider the metric tensor $M$, whose entries are given by the dot products of the respective basis vectors, ordered as follows:

$$
m_{j k}= \begin{cases}e_{j}^{+} \cdot e_{k}^{+}, & j, k=1, \ldots, m \\ e_{j}^{+} \cdot e_{k}^{-}, & j=1, \ldots, m, k=m+1, \ldots, 2 m \\ e_{j}^{-} \cdot e_{k}^{+}, & j=m+1, \ldots, 2 m, k=1, \ldots, m \\ e_{j}^{-} \cdot e_{k}^{-}, & j, k=m+1, \ldots, 2 m\end{cases}
$$


and which schematically takes the form

$$
M=\left(\begin{array}{rrrr|rrrr}
0 & -g & \cdots & -g & -\frac{1}{2} & g & \cdots & g \\
-g & 0 & \ddots & \vdots & g & -\frac{1}{2} & \ddots & \vdots \\
\vdots & \ddots & 0 & -g & \vdots & \ddots & -\frac{1}{2} & g \\
-g & \cdots & -g & 0 & g & \cdots & g & -\frac{1}{2} \\
\hline-\frac{1}{2} & g & \cdots & g & 0 & -g & \cdots & -g \\
g & -\frac{1}{2} & \ddots & \vdots & -g & 0 & \ddots & \vdots \\
\vdots & \ddots & -\frac{1}{2} & g & \vdots & \ddots & 0 & -g \\
g & \cdots & g & -\frac{1}{2} & -g & \cdots & -g & 0
\end{array}\right) .
$$

Its determinant reads

$$
\operatorname{det} M=(-1)^{m} \frac{(1+4 g)^{m-1}(1-4(m-1) g)}{4^{m}},
$$

from which we infer that in order for $M$ to be a genuine metric tensor, we should have

$$
g \neq-\frac{1}{4} \quad \text { and } \quad g \neq \frac{1}{4(m-1)},
$$

since these specific values would induce a collapse of dimension. For a further discussion on these metric aspects and their geometric interpretation, in particular in low dimension, we refer to Section 5.

Under the above conditions, $\partial^{2}$ takes the form

$$
\begin{aligned}
\partial^{2}= & -\sum_{j=1}^{m} \Delta_{j}^{+} \Delta_{j}^{-} \\
& +g \sum_{j \neq k}\left(\Delta_{j}^{+} \Delta_{k}^{-}+\Delta_{k}^{+} \Delta_{j}^{-}-\Delta_{j}^{-} \Delta_{k}^{-}-\Delta_{j}^{+} \Delta_{k}^{+}\right),
\end{aligned}
$$

where we have also rewritten the $+/-$ term in the second sum in a visually more symmetric way. The first sum exactly yields the star Laplacian $\Delta$; the second sum explicitly reads

$$
\begin{aligned}
\sum_{j \neq k}[ & \left(4 f(\mathbf{x})-f\left(\mathbf{x}+e_{j}+e_{k}\right)\right. \\
& \left.-f\left(\mathbf{x}+e_{j}-e_{k}\right)-f\left(\mathbf{x}-e_{j}+e_{k}\right)-f\left(\mathbf{x}-e_{j}-e_{k}\right)\right) \\
& +2\left(f\left(\mathbf{x}+e_{j}\right)+f\left(\mathbf{x}-e_{j}\right)+f\left(\mathbf{x}+e_{k}\right)+f\left(\mathbf{x}-e_{k}\right)\right. \\
& -4 f(\mathbf{x}))],
\end{aligned}
$$

which again splits into two terms. The second one, namely

$$
4 \sum_{j<k}\left(f\left(\mathbf{x}+e_{j}\right)+f\left(\mathbf{x}-e_{j}\right)+f\left(\mathbf{x}+e_{k}\right)+f\left(\mathbf{x}-e_{k}\right)-4 f(\mathbf{x})\right),
$$

is easily recognized as a multiple of the star Laplacian, more precisely $4(m-1) \Delta$. For the first one, namely

$$
\begin{aligned}
& -2 \sum_{j<k}\left(f\left(\mathbf{x}+e_{j}+e_{k}\right)+f\left(\mathbf{x}+e_{j}-e_{k}\right)+f\left(\mathbf{x}-e_{j}+e_{k}\right)\right. \\
& \left.+f\left(\mathbf{x}-e_{j}-e_{k}\right)-4 f(\mathbf{x})\right)
\end{aligned}
$$

we introduce the notation

$$
\begin{aligned}
\widetilde{\Delta}_{j k}= & f\left(\mathbf{x}+e_{j}+e_{k}\right)+f\left(\mathbf{x}+e_{j}-e_{k}\right)+f\left(\mathbf{x}-e_{j}+e_{k}\right) \\
& +f\left(\mathbf{x}-e_{j}-e_{k}\right)-4 f(\mathbf{x}), \quad j<k,
\end{aligned}
$$

where each $\widetilde{\Delta}_{j k}$ may be interpreted as a cross Laplacian on the corresponding $\left(e_{j}, e_{k}\right)$ plane; see also [Faustino et al. 07]. Summarizing, we have

$$
\partial^{2}=(4(m-1) g-1) \Delta-2 g \sum_{j<k} \widetilde{\Delta}_{j k},
$$

whose support is indeed contained in the closed cube with side length 2 . Note, however, that the grid points involved in the second term at the right-hand side of (3-8) are not in accordance with the originally chosen $\mathbb{Z}^{m}$ graph, which means that with respect to the rectangular frame, they are not direct neighbors of $\mathbf{x}$. Hence we will consider in the next section the particular case in which this term disappears. Anyhow, observe that if we would like to interpret (3-8) as a result of a truly similar nature to that of the continuous factorization $\partial_{\mathbf{x}}^{2}=-\Delta$, then we should restrict the metric scalar $g$ to the range $\left[0, \frac{1}{4(m-1)}[\right.$. Although we will allow for other values of $g$ as well, we want to point out the reappearance of the particular value $\frac{1}{4(m-1)}$ in this respect, a value that would cause the star Laplacian $\Delta$ to drop from (3-8).

For now, we may finally introduce the concept of discrete monogenicity.

Definition 3.5. A Clifford-algebra-valued function $f$ defined on the bounded set $B \subset \mathbb{Z}^{m}$ is called discrete monogenic in the interior of $B$ if for all $\mathbf{x} \in B$ such that also $\mathcal{N}_{\mathbf{x}} \subset B$, we have

$$
\partial[f](\mathbf{x})=0 .
$$

Defined in this way, discrete monogenicity clearly constitutes a proper generalization to higher dimension of discrete holomorphy in the Isaacs or the Ferrand sense, described above. Moreover, it may in some sense be seen as a refinement of discrete harmonicity, since the righthand side of (3-8) is interpretable as some kind of generalized discrete Laplacian, or a mixed Laplacian as it is called in [Faustino et al. 07]. Some results of the related function theory, e.g., Cauchy's theorem and a CauchyPompeiu formula, are treated in [Brackx et al. 09].

\section{A SPECIAL CASE: A FACTORIZATION OF THE STAR LAPLACIAN}

We will now consider the special case of the above approach in which $g=0$, the defining relations for the 
forward and backward basis vectors thus reducing to

$$
\begin{aligned}
e_{j}^{+}+e_{j}^{-} & =e_{j}, j=1, \ldots, m, \\
\left\{e_{j}^{+}, e_{k}^{+}\right\} & =\left\{e_{j}^{-}, e_{k}^{-}\right\}=\left\{e_{j}^{+}, e_{k}^{-}\right\}=0 \\
j, k & =1, \ldots, m, j \neq k, \\
\left(e_{j}^{+}\right)^{2} & =\left(e_{j}^{-}\right)^{2}=0, j=1, \ldots, m, \\
\left\{e_{j}^{+}, e_{j}^{-}\right\} & =-1, j=1, \ldots, m .
\end{aligned}
$$

Clearly, this particular choice for the metric scalar causes the second summand in (3-8) to drop out, whence we are left with a pure factorization of the (star) Laplace operator, i.e.,

$$
\partial^{2}=-\Delta
$$

where, moreover, the support of the involved operators not only remains in the cube with side length 2 , centered at $\mathbf{x}$, but respects the $\mathbb{Z}^{m}$ graph as well, in the sense that all involved grid points are direct neighbors of $\mathbf{x}$. In fact, there is a well-known model for these particular forward and backward vectors, namely the so-called Witt basis; see also [Faustino and Kaehler 07b].

In order to present this model properly, we consider again the Clifford algebra $\mathbb{C}_{2 m}$ in which $\mathbb{R}_{0, m}$ has been embedded, and which we will provide with the structure of a Hermitian space by introducing a complex structure $J$ on the underlying orthogonal space $\mathbb{R}^{0,2 m}$, i.e., $J \in$ $\mathrm{SO}(2 m)$ with $J^{2}=\mathbf{- 1}$. For details on the construction of a Hermitian Clifford algebra, we refer to [Brackx et al. 07a, Brackx et al. 07b]; for our purpose the following observations will suffice. Let, as before, $\left(e_{1}, \ldots, e_{m}\right)$ be the chosen orthonormal basis of $\mathbb{R}^{0, m}$ and complement it with additional vectors $\left(e_{m+1}, \ldots, e_{2 m}\right)$ in order to obtain an orthonormal basis of $\mathbb{R}^{0,2 m}$, i.e.

$$
e_{j} e_{k}+e_{k} e_{j}=-2 \delta_{j k}, \quad j, k=1, \ldots, 2 m .
$$

Then, without loss of generality, the complex structure $J$ may always be chosen in such a way that with respect to this basis, it is represented by a block diagonal matrix, i.e., it maps the $m$-dimensional subspaces respectively spanned by $\left(e_{1}, \ldots, e_{m}\right)$ and by $\left(e_{m+1}, \ldots, e_{2 m}\right)$ onto each other. Commonly used is the complex structure represented by the matrix

$$
J=\left[\begin{array}{cc}
0 & \mathbf{1}_{m} \\
-\mathbf{1}_{m} & 0
\end{array}\right],
$$

or in other words, acting on the basis vectors as

$$
J\left[e_{j}\right]=-e_{m+j} \quad \text { and } \quad J\left[e_{m+j}\right]=e_{j}, \quad j=1, \ldots, m,
$$

though other choices are possible as well. Then, the Witt basis elements $\left(\mathfrak{f}_{j}, \mathfrak{f}_{j}^{c}\right)_{j=1}^{m}$ for the complex Clifford algebra
$\mathbb{C}_{2 m}$ are obtained through the action of $\frac{1}{2}(\mathbf{1} \pm i J)$ on the orthogonal basis elements $e_{j}$ :

$$
\begin{aligned}
\mathfrak{f}_{j}=\frac{1}{2}\left(e_{j}+i J\left[e_{j}\right]\right), & j=1, \ldots, m, \\
\mathfrak{f}_{j}^{c}=\frac{1}{2}\left(e_{j}-i J\left[e_{j}\right]\right), & j=1, \ldots, m .
\end{aligned}
$$

Then $\mathfrak{f}_{j}+\mathfrak{f}_{j}^{c}=e_{j}, j=1, \ldots, m$, and it is moreover easily checked that the Witt basis elements satisfy the Grassmann identities

$$
\mathfrak{f}_{j} \mathfrak{f}_{k}+\mathfrak{f}_{k} \mathfrak{f}_{j}=\mathfrak{f}_{j}^{c} \mathfrak{f}_{k}^{c}+\mathfrak{f}_{k}^{c} \mathfrak{f}_{j}^{c}=0, \quad j, k=1, \ldots, m,
$$

which also implies their isotropy $\left(\mathfrak{f}_{j}^{2}=\left(\mathfrak{f}_{j}^{c}\right)^{2}=0, j=\right.$ $1, \ldots, m)$, and the duality identities

$$
\mathfrak{f}_{j} \mathfrak{f}_{k}^{c}+\mathfrak{f}_{k}^{c} \mathfrak{f}_{j}=-\delta_{j k}, \quad j, k=1, \ldots, m
$$

This exactly coincides with the above conditions on the vectors $e_{j}^{+}$and $e_{j}^{-}$, so that we may put $e_{j}^{+}=\mathfrak{f}_{j}$ and $e_{j}^{-}=\mathfrak{f}_{j}^{c}, j=1, \ldots, m$, and we are left with the Witt discrete Dirac operator $\partial=\partial^{+}+\partial^{-}$, with

$$
\partial^{+}=\sum_{j=1}^{m} \mathfrak{f}_{j} \Delta_{j}^{+} \quad \text { and } \quad \partial^{-}=\sum_{j=1}^{m} \mathfrak{f}_{j}^{c} \Delta_{j}^{+},
$$

for which the factorization (3-8) holds. Although this setting was already mentioned in [Novikov 99], to the authors' knowledge, no discrete function theory has yet been developed for this operator; for some first results we refer to [Brackx et al. 09].

\section{A MODEL FOR THE FORWARD AND BACKWARD BASIS VECTORS}

In Section 3 we introduced a discrete Dirac operator with respect to the $\mathbb{Z}^{m}$ graph, the square of which is a scalar second-order difference operator, which may be interpreted as a kind of discrete Laplace operator (a mixed Laplacian) with support contained in the cube with side length 2 , centered at the point $\mathbf{x}$ under consideration. A crucial role is played by the forward and backward Clifford basis vectors $e_{j}^{+}$and $e_{j}^{-}, j=1, \ldots, m$, for which, up to now, we have provided only a concrete model in the special case above. Hence our attention is now directed toward the construction of a feasible model for them in the general case that the metric scalar $g$ is not equal to zero.

To this end, and inspired by the definition of the Witt basis elements in the previous section, we introduce curvature vectors $B_{j}, j=1, \ldots, m$, and we put, quite 
symmetrically,

$$
\begin{aligned}
& e_{j}^{+}=\frac{1}{2}\left(e_{j}+B_{j}\right), \quad j=1, \ldots, m, \\
& e_{j}^{-}=\frac{1}{2}\left(e_{j}-B_{j}\right), \quad j=1, \ldots, m,
\end{aligned}
$$

so that already $e_{j}^{+}+e_{j}^{-}=e_{j}, j=1, \ldots, m$. We now want the forward and backward vectors defined in this way to satisfy the relations derived in the foregoing sections. Firstly, in order to ensure their isotropy, we should have

$$
e_{j}^{2} \pm\left\{e_{j}, B_{j}\right\}+B_{j}^{2}=0, \quad j=1, \ldots, m,
$$

or equivalently,

$$
\begin{aligned}
B_{j}^{2} & =+1, \\
\left\{e_{j}, B_{j}\right\} & =2\left(e_{j} \cdot B_{j}\right)=0, \quad j=1, \ldots, m .
\end{aligned}
$$

Under these conditions the anticommutation relation $\left\{e_{j}^{+}, e_{j}^{-}\right\}=-1, j=1, \ldots, m$, is seen to be satisfied as well. Next, we express the other anticommutation rules as well: from $\left\{e_{j}^{+}, e_{k}^{+}\right\}=\left\{e_{j}^{-}, e_{k}^{-}\right\}=-2 g$ we learn that

$$
\begin{gathered}
\pm\left\{e_{k}, B_{j}\right\} \pm\left\{e_{j}, B_{k}\right\}+\left\{B_{j}, B_{k}\right\}=-8 g, \\
j, k=1, \ldots, m, j \neq k, \text { while }\left\{e_{j}^{+}, e_{k}^{-}\right\}=2 g \text { implies } \\
\left\{e_{k}, B_{j}\right\}-\left\{e_{j}, B_{k}\right\}-\left\{B_{j}, B_{k}\right\}=8 g, \\
j, k=1, \ldots, m, j \neq k, \text { or equivalently } \\
\left\{e_{k}, B_{j}\right\}=2\left(e_{k} \cdot B_{j}\right)=0, \\
\left\{B_{k}, B_{j}\right\}=2\left(B_{k} \cdot B_{j}\right)=-8 g \quad j, k=1, \ldots, m, j \neq k .
\end{gathered}
$$

Note that the second condition in (5-1) and the first one in (5-2) together express the orthogonality of the space spanned by the curvature vectors and the original $m$ dimensional space with basis $\left(e_{1}, \ldots, e_{m}\right)$. As a consequence, also taking into account that $B_{j}^{2}=+1$, we may write the curvature vectors explicitly as

$$
B_{j}=\sum_{\ell=1}^{m} b_{j}^{(\ell)}\left(i e_{m+\ell}\right)=\sum_{\ell=1}^{m} b_{j}^{(\ell)} \epsilon_{\ell}, \quad j=1, \ldots, m,
$$

where

$$
\epsilon_{\ell}^{2}=\left(i e_{m+\ell}\right)^{2}=+1, \quad \ell=1, \ldots, m,
$$

and

$$
\sum_{\ell=1}^{m}\left(b_{j}^{(\ell)}\right)^{2}=1, \quad j=1, \ldots, m .
$$

Note that here the Clifford dot product of any two curvature vectors equals their Euclidean inner product:

$$
B_{k} \cdot B_{j}=\left\langle B_{k}, B_{j}\right\rangle=\sum_{\ell=1}^{m} b_{k}^{(\ell)} b_{j}^{(\ell)}, \quad j, k=1, \ldots, m .
$$

These inner products all being equal to the same scalar $-4 g$, we may thus interpret $B_{1}, \ldots, B_{m}$ as a set of vectors on the unit sphere $S^{m-1}$ of $\mathbb{R}^{m}$, containing pairwise the same fixed angle $\alpha$, with $\cos (\alpha)=-4 g$. Note that in order for this to be possible, we need to restrict $g$ to the interval $\left.]-\frac{1}{4}, \frac{1}{4}\right]$. Then we may see the set of curvature vectors as a kind of "umbrella" that will open and close according to varying $g$. In particular, for $g=0$ we obtain $\alpha=\frac{\pi}{2}$, in agreement with the Witt case of the previous section.

The above relations (5-1)-(5-2) are summarized in the metric tensor $\widetilde{M}$ :

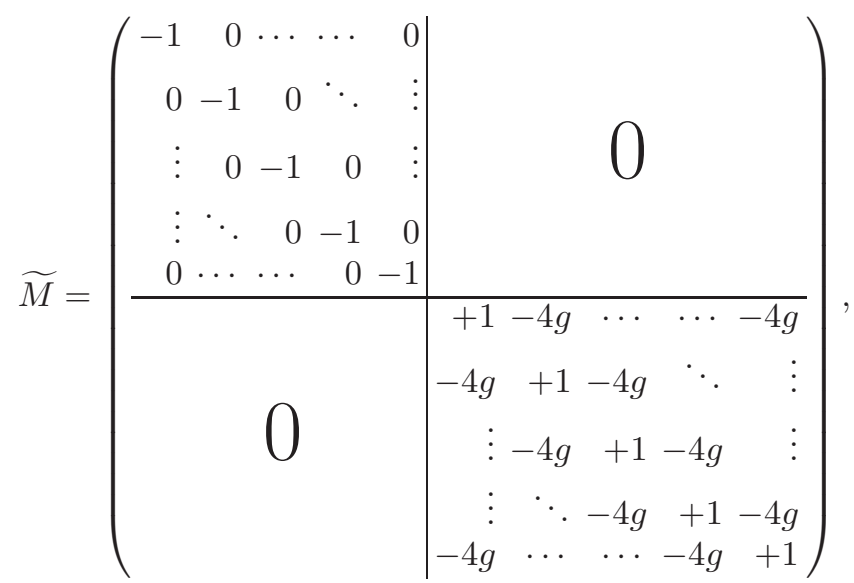

with entries equal to the Clifford dot products of all vectors under consideration, namely $\left(e_{1}, \ldots, e_{m}, B_{1}, \ldots, B_{m}\right)$, in this specific order. Its determinant reading

$$
\operatorname{det} \widetilde{M}=(-1)^{m}(1+4 g)^{m-1}(1-4(m-1) g),
$$

we are again confronted with the inadmissible values $-\frac{1}{4}$ and $\frac{1}{4(m-1)}$ for the metric scalar $g$, as already obtained in Section 3. Indeed, in those cases we no longer dispose of a basis for a $2 m$-dimensional space: instead, for $g=\frac{1}{4(m-1)}$ we have that $\operatorname{rank}(\widetilde{M})=2 m-1$, while for $g=-\frac{1}{4}$ we even obtain $\operatorname{rank}(\widetilde{M})=m+1$. We will now further investigate this collapse of dimension from a geometric point of view.

To this end, first take $g=-\frac{1}{4}$. Here we have that

$$
B_{k} \cdot B_{j}=\left\langle B_{k}, B_{j}\right\rangle=+1, \quad j, k=1, \ldots, m,
$$

whence their contained angle $\alpha$ becomes zero. So, the "umbrella" described above completely closes, all curvature vectors coincide, and the dimension of the space spanned by them reduces to 1 , which is in accordance with the rank of the metric tensor $\widetilde{M}$, or in other words the dimension of the overall space, equaling $m+1$. 
In the second case, i.e., $g=\frac{1}{4(m-1)}$, the rank of $\widetilde{M}$ shows that the space spanned by the curvature vectors should be $(m-1)$-dimensional, i.e., they should be on the intersection of the unit sphere $S^{m-1}$ with a hyperplane in $m$-dimensional space. For low dimension, say $m=3$ and $m=4$, we will determine their contained angle in that particular situation and show that it exactly corresponds to the given value of $g$.

So let $m=3$, let $g$ be arbitrary again, and put, in spherical coordinates,

$$
B_{k}=\left[\sin \left(\varphi_{k}\right) \sin \left(\theta_{k}\right), \cos \left(\varphi_{k}\right) \sin \left(\theta_{k}\right), \cos \left(\theta_{k}\right)\right],
$$

$k=1,2,3$, with $\varphi_{k} \in\left[0,2 \pi\left[\right.\right.$ and $\theta_{k} \in[0, \pi[$. A symmetry argument leads us to assume that the angles $\theta_{k}$ with the $z$-axis are all equal, i.e.,

$$
\theta_{k}=\theta, \quad k=1,2,3
$$

the Euclidean inner product of two curvature vectors thus taking the form

$-4 g=\cos (\alpha)=\left\langle B_{k}, B_{l}\right\rangle=\cos ^{2}(\theta)+\sin ^{2}(\theta) \cos \left(\varphi_{k}-\varphi_{l}\right)$,

$k, l=1,2,3$. Observe that $\theta=0$ corresponds to the already described situation in which the three curvature vectors coincide (and are equal to $[0,0,1]$ ), which can be seen either from (5-3) or (5-4), the latter also showing that in that case $\cos (\alpha)=1$ and $g=-\frac{1}{4}$, thus confirming the above result. On the other hand, the current situation of interest, with the three curvature vectors spanning a plane, is now easily seen to correspond to $\theta=\frac{\pi}{2}$, the plane then simply being $z=0$. In general, from (5-4) it also follows that $\cos \left(\varphi_{k}-\varphi_{l}\right)$ should be independent of $k$ and $l$, so that we may put

$$
\varphi_{k}=(k-1) \frac{2 \pi}{3}, \quad k=1,2,3,
$$

independent of $g$, in this way ensuring in all cases a symmetric position of the curvature vectors with respect to the $z$-axis. The choices made cause (5-4) to reduce to

$$
-4 g=\cos (\alpha)=\cos ^{2}(\theta)-\frac{1}{2} \sin ^{2}(\theta),
$$

from which we learn that $\theta=\frac{\pi}{2}$ corresponds to $\cos (\alpha)=$ $-\frac{1}{2}$ and $g=\frac{1}{8}$, in this way indeed recovering the predicted value $g=\frac{1}{4(m-1)}$, here with $m=3$. The curvature vectors then explicitly read

$B_{1}=[0,1,0], \quad B_{2}=\left[\frac{\sqrt{3}}{2},-\frac{1}{2}, 0\right], \quad B_{3}=\left[-\frac{\sqrt{3}}{2},-\frac{1}{2}, 0\right]$.
They are uniquely determined up to an arbitrary rotation around the $z$-axis. Also observe that $(5-5)$ now allows us to calculate, for any given (yet admissible) $g$, the corresponding position of the curvature vectors.

Next, let $m=4$. In spherical coordinates, we now have, for $k=1,2,3,4$,

$$
\begin{aligned}
B_{k}=[ & \sin \left(\varphi_{k}\right) \sin \left(\theta_{k}\right) \sin \left(\chi_{k}\right), \cos \left(\varphi_{k}\right) \sin \left(\theta_{k}\right) \sin \left(\chi_{k}\right), \\
& \left.\cos \left(\theta_{k}\right) \sin \left(\chi_{k}\right), \cos \left(\chi_{k}\right)\right] .
\end{aligned}
$$

By a similar symmetry argument as above, we are led to assume equal angles $\chi_{k}$, i.e., $\chi_{k}=\chi$, and equidistant angles $\varphi_{k}$, i.e., $\varphi_{k}=(k-1) \frac{\pi}{2}, k=1,2,3,4$. The Euclidean inner product of any two curvature vectors may thus be written as

$$
\left\langle B_{k}, B_{l}\right\rangle=\cos ^{2}(\chi)+\sin ^{2}(\chi) \cos \left(\theta_{k}\right) \cos \left(\theta_{l}\right)
$$

when $\{k, l\}=\{1,2\},\{2,3\},\{3,4\}$, or $\{4,1\}$, and as

$$
\left\langle B_{k}, B_{l}\right\rangle=\cos ^{2}(\chi)+\sin ^{2}(\chi) \cos \left(\theta_{k}+\theta_{l}\right)
$$

when $\{k, l\}=\{1,3\}$ or $\{2,4\}$. Since all these expressions should be equal, further calculations yield that

$$
\theta_{3}=\theta_{1}=\theta \quad \text { and } \quad \theta_{4}=\theta_{2}=\pi-\theta
$$

with

$$
\theta=\arccos \left(\frac{1}{\sqrt{3}}\right)
$$

whence both (5-6) and (5-7) lead to

$$
-4 g=\cos (\alpha)=\cos ^{2}(\chi)-\frac{1}{3} \sin ^{2}(\chi) .
$$

Note that all occurring angles, except for the remaining angle $\chi$, have been determined independently of $g$. It is only through $\chi$ and (5-8) that a direct relation is obtained between the metric scalar $g$, the contained angle $\alpha$ of any two curvature vectors, and their explicit position on the unit sphere. As above, two special cases are of interest. First of all, for $\chi=0$, and correspondingly $\alpha=0$ and $g=\frac{1}{4}$, we are again confronted with the situation in which all curvature vectors coincide. Next, also for $\chi=\frac{\pi}{2}$, the curvature vectors become linearly dependent: indeed, denoting the Cartesian coordinates by $[x, y, z, w]$, they will all belong to the hyperplane $w=0$. Here, $\cos (\alpha)$ assumes the value $-\frac{1}{3}$, from which we arrive at $g=\frac{1}{12}$, which is equal to $\frac{1}{4(m-1)}$ with $m=4$, as expected.

\section{ACKNOWLEDGMENTS}

Financial support of the FWO Vlaanderen, by a special grant ("Krediet aan navorsers" no. 31506208) for research on the subject of discrete Clifford analysis, is gratefully acknowledged by Frank Sommen. 


\section{REFERENCES}

[Benjamini and Lovász 02] I. Benjamini and L. Lovász. "Harmonic and Analytic Functions on Graphs." J. Geom. 76 (2002), 3-15.

[Brackx et al. 82] F. Brackx, R. Delanghe, and F. Sommen. Clifford Analysis. Boston: Pitman, 1982.

[Brackx et al. 07a] F. Brackx, J. Bureš, H. De Schepper, D. Eelbode, F. Sommen, and V. Souček. "Fundaments of Hermitean Clifford Analysis. Part I: Complex Structure." Comp. Anal. Oper. Theory 1:3 (2007), 341-365.

[Brackx et al. 07b] F. Brackx, J. Bureš, H. De Schepper, D. Eelbode, F. Sommen, and V. Souček. "Fundaments of Hermitean Clifford Analysis. Part II: Splitting of $h$-Monogenic Equations." Comp. Var. Elliptic Equ. 52:10-11 (2007), 1063-1079.

[Brackx et al. 09] F. Brackx, H. De Schepper, F. Sommen, and L. Van de Voorde. "Discrete Clifford Analysis: A Germ of Function Theory." In Hypercomplex Analysis, edited by I. Sabadini, M. Shapiro, and F. Sommen, pp. 37-54, Trends in Mathematics. Boston: Birkhäuser, 2009.

[Delanghe et al. 92] R. Delanghe, F. Sommen, and V. Souček. Clifford Algebra and Spinor-Valued Functions. Dordrecht: Kluwer Academic Publishers, 1992.

[Duffin 56] R. Duffin. "Basic Properties of Discrete Analytic Functions." Duke Math. J. 23 (1956), 335-363.

[Faustino and Kaehler 07a] N. Faustino and U. Kaehler. "Fischer Decomposition for Difference Dirac Operators." Adv. Appl. Clifford Algebras 17:1 (2007), 37-58.

[Faustino and Kaehler 07b] N. Faustino and U. Kaehler. "On a Correspondence Principle between Discrete Differential Forms, Graph Structure and Multi-vector Calculus on Symmetric Lattices." arXiv:0712.1004, 2007.

[Faustino et al. 06] N. Faustino, K. Guerlebeck, A. Hommel, and U. Kaehler. "Difference Potentials for the NavierStokes Equations in Unbounded Domains." J. Difference Equ. Appl. 12:6 (2006), 577-595.

[Faustino et al. 07] N. Faustino, U. Kaehler, and F. Sommen. "Discrete Dirac Operators in Clifford Analysis." Adv. Appl. Clifford Algebras 17:3 (2007), 451-467.
[Ferrand 44] J. Ferrand. "Fonctions préharmoniques et fonctions préholomorphes." Bull. Sci. Math. 68 (1944), 152180.

[Gilbert and Murray 91] J. Gilbert and M. Murray. Clifford Algebras and Dirac Operators in Harmonic Analysis. Cambridge: Cambridge University Press, 1991.

[Guerlebeck and Hommel 01] K. Guerlebeck and A. Hommel. "On Finite Difference Dirac Operators and Their Fundamental Aolutions." Adv. Appl. Clifford Algebras 11:S2 (2001), 89-106.

[Guerlebeck and Hommel 02] K. Guerlebeck and A. Hommel. "On Finite Difference Potentials and Their Applications in a Discrete Function Theory." Math. Methods Appl. Sci. 25:16-18 (2002), 1563-1576.

[Guerlebeck and Sproessig 90] K. Guerlebeck and W. Sproessig. Quaternionic Analysis and Elliptic Boundary Value Problems, International Series of Numerical Mathematics 89. Basel: Birkhäuser, 1990.

[Guerlebeck and Sproessig 97] K. Guerlebeck and W. Sproessig. Quaternionic and Clifford Calculus for Engineers and Physicists. Chichester: John Wiley \& Sons, 1997.

[Isaacs 52] R. P. Isaacs. "Monodiffric Functions." In Construction and Applications of Conformal Maps, Proceedings of a Symposium, pp. 257-266, National Bureau of Standards Appl. Math. Ser. 18. Washington, D.C.: U.S. Government Printing Office, 1952.

[Kenyon 00] R. Kenyon. "Conformal Invariance of Domino Tiling." Ann. Probab. 28 (2000), 759-795.

[Kiselman 05] C. O. Kiselman. "Functions on Discrete Sets Holomorphic in the Sense of Isaacs, or Monodiffric Functions of the First Kind." Sci. China Ser. A 48 (2005), 86-96.

[Nakamura and Rosenfeld 97] A. Nakamura and A. Rosenfeld. "Digital Calculus." Inform. Sci. 98:1-4 (1997), 83-98.

[Novikov 99] S. P. Novikov. "Schrödinger Operators on Graphs and Symplectic Geometry." In The Arnoldfest, edited by E. Bierstone, B. Khesin, A. Khovanskii, and J. Marsden, pp. 397-413, Fields Institute Communications 24. Providence, RI: American Math. Soc., 1999.

[Porteous 95] I. Porteous. Clifford Algebras and the Classical Groups, Cambridge Studies in Advanced Mathematics 50. Cambridge: Cambridge University Press, 1995.

Hennie De Schepper, Clifford Research Group, Department of Mathematical Analysis, Faculty of Engineering, Ghent University, Galglaan 2, 9000 Gent, Belgium (hennie.deschepper@ugent.be)

Frank Sommen, Clifford Research Group, Department of Mathematical Analysis, Faculty of Sciences, Ghent University, Galglaan 2, 9000 Gent, Belgium (fs@cage.ugent.be)

Liesbet Van de Voorde, Clifford Research Group, Department of Mathematical Analysis, Faculty of Engineering, Ghent University, Galglaan 2, 9000 Gent, Belgium (liesbet.vandevoorde@ugent.be)

Received July 29, 2008; accepted February 17, 2009. 